Rev. salud pública. 6 (2):167-182, 2004

\title{
Perímetros Braquial y Cefálico como Indicadores de Pobreza y Enfermedad Diarréica Aguda en Niños Menores de 5 Años, en Bogotá
}

\author{
Ricardo Sánchez ${ }^{\mathrm{I}}$ Jairo Echeverry ${ }^{\mathrm{II}}$, Rodrigo Pardo ${ }^{\mathrm{II}}$ \\ I Médico. Especialista en Psiquiatría. Especialista en Estadística. M. Sc. \\ Epidemiología Clínica.. Facultad de Medicina, Universidad Nacional de Colombia. \\ E-Mail: rsanchezpe@unal.edu.co. \\ II Médico. Especialista en Pediatría. M. Sc. (candidato). Epidemiología Clínica. \\ Facultad de Medicina, Universidad Nacional de Colombia. E-mail: \\ jecheverryr@unal.edu.co \\ III Médico. Especialista en Neurología. M. Sc. (c) Epidemiología clínica. Facultad \\ de Medicina, Universidad Nacional de Colombia. E-mail: rpardot@unal.edu.co
}

Recibido 16 Febrero 2004/Enviado para Modificación 28 Mayo 2004/Aceptado 4 Junio 2004

\section{RESUMEN}

Objetivo Evaluar la relación entre estado nutricional y algunos factores relacionados con pobreza (diarrea aguda y hacinamiento) en niños menores de 6 años en Bogotá.

Métodos Se efectuó un estudio transversal de base comunitaria, en el año 2000 en el área urbana de Bogotá, usando una encuesta de hogares. Participaron 3081 adultos que tenían en sus hogares menores de 5 años. Este tamaño muestral se estableció para determinar un parámetro poblacional binomial. Se efectuó un muestreo multietápico. El número total de la muestra se redujo a 2833 por borrado de observaciones con valores faltantes en las variables dependientes (VDs). Los perímetros cefálico y braquial fueron tomados como proxy del estado nutricional. Se efectuó un MANOVA 2x2x2 sobre las dos VDs. Las variables independientes fueron hacinamiento (como medida de marginalización) e infección intestinal aguda reciente. El análisis incluyó ajuste secuencial por no ortogonalidad.

Resultados Las VDs mostraron baja correlación $(r=0.3)$ y distribución simétrica. La evaluación de las premisas de linearidad y multicolinearidad fueron satisfactorias. El test de ómnibus, usando el criterio de Wilk, mostró que las VDs combinadas resultaban significativamente afectadas por diarrea aguda reciente $(F=9.65 p=0.000)$, por edad menor de un año $(F=350.81 p=0.000)$ 
y por la interacción diarrea aguda-hacinamiento $(F=6.25 \mathrm{p}=0.000)$. Para evaluar la repercusión de cada efecto principal sobre las VDs individuales se efectuó un análisis de reducción de Roy-Bargmann. La homogeneidad de la regresión fue alcanzada por todos los componentes del análisis de reducción. El perímetro braquial tiene una contribución única para predecir diarrea aguda reciente (Roy-Bargmann $\mathrm{F}_{1,1}=13.1 \mathrm{p}=0.000$ ).

El perímetro cefálico tiene una contribución única para predecir diarrea aguda junto con hacinamiento (Roy-Bargmann $F_{1,1}=10.22 \mathrm{p}=0.001$ ).

Conclusiones Los episodios recientes de diarrea y la pobreza, se relacionan con pobre condición nutricional. Los perímetros cefálico y braquial podrían ser indicadores atractivos de la condición nutricional subyacente.

Palabras Claves: Pobreza, diarrea, niños, Bogotá (fuente: DeCS, BIREME).

\section{ABSTRACT}

The brachial and cephalic perimeters as indicators of poverty and acute diarrhea in children under five years in Bogotá

Objective To assess the relationship between nutritional status and some factors related with poverty (acute diarrhea and overcrowding) in children less than 6 years of age in Bogotá

Methods A community-based, cross-sectional study, was conducted during 2000 in the urban area of Bogotá, using a household interview survey. 3081 adults having in their homes children under five years were interviewed. This number of participants was calculated to obtain a representative sample of the city for estimating a binomial population parameter. A multistage sampling was carried out. The sample size was reduced to 2833 with the elimination of cases missing scores on dependent variables (DVs). Cephalic and brachial perimeters (continuous variables), used as a proxy of nutritional status (DVs), were selected as the main outcome measures. A $2 \times 2 \times 2$ multivariate analysis of variance was performed on two DVs. Independent variables were overcrowding (as a measure of marginalization) and recent acute diarrhea. The analyses included sequential adjustment for nonorthogonality.

Results DVs showed a low correlation $(r=0.3)$ and a symmetric distribution. Evaluation of assumptions of linearity and multicollinearity were satisfactory. The omnibus test, using Wilk's criterion, showed that combined DVs were significantly affected by recent acute diarrhea $(F=9.65 p=0.000)$, age less than 1 year $(F=350.81 \mathrm{p}=0.000)$ and the interaction acute diarrhea - overcrowding ( $F=6.25 p=0.000)$. In order to evaluate the impact of each main effect on the individual DVs, a Roy-Bargmann stepdown analysis was carried out. Homogeneity of regression was achieved for all components of the stepdown analysis. The brachial perimeter has a unique contribution to predict recent acute diarrhea (Roy-Bargmann $F_{1,1}=13.1 p=0.000$ ). The cephalic perimeter has a unique contribution to predict acute diarrhea and overcrowding (Roy-Bargmann $\mathrm{F}_{1,1}=10.22 \mathrm{p}=0.001$ ). 
Conclusions Recent acute diarrhea and overcrowding are related with poor nutritional status. Brachial and cephalic perimeters could be attractive indicators of different varieties of nutritional deficiency.

Key Words: Poverty, diarrhea, child, Bogotá (source: MeSH, NLM).

$\mathrm{E}$ xiste una intensa interrelación entre enfermedad diarreica aguda (EDA), desnutrición (DNT) y pobreza (1-3). La EDA, la DNT, la infección respiratoria aguda (IRA), el sarampión y el paludismo, dan cuenta de 7 de cada diez muertos, en niños menores de 5 años en los países en vías de desarrollo y por ello son las enfermedades prevalentes en la infancia, agrupadas como objeto de trabajo para su disminución a través de iniciativas y programas mundiales como la estrategia AIEPI (Atención Integrada de las Enfermedades Prevalentes de la Infancia). En los sitios donde estos eventos son emergentes, prevalentes o endémicos, existe un común denominador: pobreza y precarias condiciones de vida (4).

La EDA ha sido definida como la presencia de 3 o más deposiciones en 24 horas $(5,6)$. En términos generales es una entidad de origen infeccioso, autolimitada y que en su forma aguda dura por término medio ocho días (7). La EDA es un triste patrimonio de las regiones donde falta el agua potable, no se dispone de adecuada eliminación de excretas y donde hay hacinamiento, malas condiciones sanitarias y déficit nutricional (8).

La pobreza ha sido definida, desde la perspectiva estatal como la ausencia en la cobertura adecuada de las necesidades elementales para la subsistencia humana (9). Colombia adoptó y elaboró, desde 1 987, dos metodologías en el intento de obtener estimadores de la proporción de personas pobres y analizar algunas de sus características sociodemográficas. Las dos metodologías señaladas, son las de Necesidades Básicas Insatisfechas (NBI) y la de Línea de Pobreza (LP).

La primera metodología capta los hogares que tienen carencias en bienes y servicios que se consideran esenciales para la subsistencia de sus miembros. La metodología de LP capta los hogares que tienen un ingreso por debajo del que se considera como mínimo requerido para adquirir un conjunto de bienes y servicios necesarios para el sustento de sus miembros. Estas metodologías han sido utilizadas ampliamente en otros países del entorno latinoamericano (10). Los indicadores NBI son: Hogares con vivienda inadecuada (según sus pisos y paredes), Hogares sin servicios básicos (sin agua ni acueducto), Hogares con hacinamiento crítico (más de tres personas 
por cuarto de dormitorio), Hogares con inasistencia escolar (al menos un niño entre 6 y 12 años sin acudir a la escuela), Hogares con alta dependencia económica (jefe de hogar desempleado, o con más de tres personas a su cargo y con un nivel de escolaridad inferior a segundo primaria).

Recientemente, se ha comentado que los indicadores NBI no son adecuadas para medir la situación de pobreza en ciudades capitales como Bogotá D.C; sin embargo los indicadores siguen siendo vigentes (11).

La malnutrición es un desorden en el que están alteradas la estructura y la función orgánicas, como consecuencia de una deficiencia o un exceso en la utilización de nutrientes a escala tisular. Desde esta perspectiva la malnutrición incluye dos tipos extremos de presentación: desnutrición o sobre-nutrición (obesidad). La desnutrición entonces podría ser definida como la consecuencia de un incompleto, inadecuado, insuficiente o desequilibrado uso, utilización o disposición de nutrientes a nivel de los tejidos (12).

Puede a su vez clasificarse cualitativamente (13) según el origen de ella (primaria o secundaria), el tiempo de evolución (aguda, crónica), su gravedad (leve, moderada, severa), o su expresión, dependiendo el tipo de nutriente global (proteico-calórico o Kwashiorkor) o nutriente circunstancial (vitamina, mineral u oligoelemento) insuficiente en la economía.

El estado nutricional de los niños, se ha relacionado más con las infecciones que con la disponibilidad de comida (14). Esto podría significar que el tratamiento primario de toda desnutrición no es el soporte aislado de alimento, sino la promoción de la salud y la prevención o el tratamiento de las infecciones de la infancia.

Suficiente evidencia relaciona la Enfermedad Diarreica Aguda (EDA) con la Desnutrición (DNT) en la población infantil $(15,16)$. Esta relación cumple con los criterios de la causalidad (17) (fuerza, consistencia, especificidad, gradiente biológico, plausibilidad, coherencia, analogía) excepto en lo referente a la relación temporal. Esto es, no puede establecerse cuál variable precedió a la otra en el tiempo, si bien se ha considerado tradicionalmente que la consecuencia nutricional de la EDA es la DNT proteico - calórica y el retardo en el crecimiento $(18,19)$.

Así las cosas, la relación existente es de índole bi-direccional o sea que la EDA puede llevar a DNT y viceversa, pudiéndose perpetuar en un círculo vicioso cuando quiera que alguna de las dos variables se presenten de manera intensa, persistente o recurrente (20). La DNT y la EDA son dos varia- 
bles del anfitrión, pero que dependen intensamente del medio ambiente, muy especialmente de saneamiento ambiental e intra-domiciliario, pobreza, hacinamiento, falta de higiene, analfabetismo y otros factores socio-culturales de conocimientos, actitudes y prácticas (21).

Los niños desnutridos tienden a tener infecciones por largos períodos y suelen dar manifestaciones clínicas más severas de la enfermedad (20). En ese mismo sentido, en las áreas geográficas donde más existe DNT, hay una mayor densidad de incidencia de EDA y de enfermedades prevalentes.

Los niños con EDA tienen deprimido el apetito y esto es más marcado en la medida en que el cuadro gastrointestinal es más intenso (18). El cuadro diarreico es en sí un estado de malabsorción agudo, con la consiguiente pérdida de líquidos, electrolitos y nutrientes, el cual, con la hiporexia existente, determina finalmente un episodio de desplome nutricional de variable intensidad $(18,19)$. Los estudios han demostrado que el período inmediatamente subsiguiente al cese del episodio diarreico es el momento ideal (y no otro) para la recuperación de la condición nutricional preexistente a dicho episodio (14). La falta de recuperación suficiente de las pérdidas incurridas durante la convalecencia, determina un saldo en rojo nutricional y una pérdida de las oportunidades para alcanzar el crecimiento genético potencial es un hecho demostrado hace más de 50 años (22).

Es muy laborioso y habitualmente difícil la evaluación o determinación de desnutrición en un paciente, excepto en situaciones extremas. La fase clínica de la enfermedad (donde se hacen evidentes síntomas y signos derivados del elemento insuficiente) es tardía en la historia natural de ella.

El problema radica, desde cierta perspectiva, en una ausencia de un estándar de referencia adecuado, posiblemente por la naturaleza hiper-compleja y multifactorial en causas y efectos que subyacen a ella. La mayoría de los parámetros investigados se han basado en su distribución "normal" estadística, sin poder asegurar con certidumbre si aquellos individuos cuyos valores se encuentran incluidos dentro de los parámetros normales son nutridos, y por el contrario, aquellos por fuera de ellos son malnutridos.

De cualquier forma, tradicionalmente se han utilizado la anamnesis general y alimentaria y el examen físico como elementos diagnósticos iniciales de la situación nutricional (23). En términos prácticos, el indicador más importante desde el punto de vista funcional para la determinación del estado nutricional en pediatría es la velocidad de crecimiento pondo-estatural (24). Con base en mediciones antropométricas se han propuesto diferentes escalas 
para su determinación en forma categórica, como el peso para la edad, el peso para la talla (25), o el peso para la talla en razón de la edad (26).

Como lo referíamos previamente, estas escalas funcionan adecuadamente en condiciones extremas, pero presentan grandes inconsistencias en el momento de evaluar niños con sospecha clínica de malnutrición (27).

El problema que subyace en este aspecto es la escasa existencia de parámetros locales o regionales de las medidas de crecimiento pondo-estatural y de velocidad de crecimiento infantil. Dada la falta de evidencia sólida que apoye las recomendaciones, en forma reciente la Organización Mundial de la Salud (OMS) ha propuesto adoptar en todos los países (desarrollados o no) las curvas que con base en una distribución percentilar fueron elaboradas por el Centro Nacional de Estadística de Salud de los Estados Unidos (NCHS) (28). Se deben ajustar para edad las mediciones de peso encontradas mediante un proceso de estandarización $\mathrm{Z}$, y determinar la severidad de la desnutrición de acuerdo con las disminuciones progresivas en los valores negativos de las desviaciones estándar obtenidas (p.ej. -2, -3,-4, etc). A partir de menos dos ( -2 DS) se considera el punto de corte para definir desnutrición (29).

Sin embargo, surge el inconveniente de reproducir el proceso en áreas donde no se dispone de cartas o tablas, o donde claramente las tablas de la NCHS no serían apropiadas.

Recientemente se ha utilizado la medición del perímetro del brazo en su punto medio, como indicador nutricional. Es atractivo por cuanto es fácil, puede hacerlo cualquier observador, no necesariamente del área de la salud, sin experiencia especifica, con elementos muy rudimentarios, de manera reproducible y sin la necesidad de acudir a una escala o cartilla que puede no estar disponible en el momento en que se necesite. Tiene poca variabilidad entre el año y los 5 años de edad del niño. En este rango, mediciones de $12,5 \mathrm{~cm}$ a $13,5 \mathrm{~cm}$ se consideran compatibles con DNT moderada y cifras inferiores a 12,5 DNT severa (30). La Fundación CIMDER de la Universidad del Valle validó una cinta de colores, que al medir el perímetro del brazo, estima de manera rápida y reproducible la condición nutricional de niños menores de 6 años (31).

Dadas las interacciones existentes entre EDA, pobreza y DNT, y las dificultades inherentes al establecimiento de la condición nutricional, este trabajo intenta establecer la relación existente entre la presencia de EDA reciente, el hacinamiento (como variable proxi del estado de pobreza) y los pe- 
rímetros braquial y cefálico (como variables proxi somatométricas del estado nutricional) de los niños menores de 5 años de Bogotá.

Si existiere alguna relación entre la medición del perímetro del brazo o el cefálico, con la aparición de un evento de diarrea y pobreza, estas medidas antropométricas podrían caracterizar más adecuadamente a aquellos individuos en estado de pobreza y en mayor riesgo de EDA o viceversa.

\section{PACIENTES, MATERIALES Y MÉTODOS}

Los datos corresponden a una encuesta poblacional efectuada el año 2000 en Bogotá, dentro de un proyecto para evaluar los programas de enfermedad diarreica aguda (EDA) e infección respiratoria aguda (IRA). En dicho estudio se empleó el modelo de Donabedian (32) para evaluación de calidad de atención. Como población de estudio del componente comunitario de dicha evaluación, se consideraron los hogares, los cuidadores y niños y niñas menores de cinco años de edad. De dicha población se tomó una muestra probabilística por medio de un diseño de muestreo multietápico, una de cuyas fases implicó estratificación con asignación proporcional al tamaño del estrato. Para efecto de la precisión de estimadores requeridos en dicho estudio se determinó un tamaño muestral de 3264 unidades de observación. En esta muestra se aplicó una encuesta de hogares para explorar conocimientos, actitudes y prácticas frente a EDA. La existencia de hacinamiento crítico y la presencia de al menos un episodio de EDA en los últimos 6 meses, se verificó mediante la pregunta directa al informante cuidador. Simultáneamente, personal entrenado, efectuó en los niños de tales hogares la medición de los perímetros cefálico y braquial (tercio medio, brazo derecho) de acuerdo a los estándares $(24,25,26,27)$. Fueron descartados para el análisis aquellos niños con trastornos evidentes del Sistema Nervioso Central o con lesiones agudas o recientes en los miembros superiores. En el presente artículo se seleccionaron aquellas observaciones en las cuales las encuestas y las mediciones antropométricas aportaran información completa en todas las variables requeridas para el análisis (2 845 encuestas).

Asumiendo que unos indicadores sencillos para medir el estado nutricional son el perímetro cefálico y el perímetro braquial, se tomaron estas mediciones como variables dependientes. Como variables independientes, que pueden afectar los valores de las variables dependientes, se tomaron las siguientes: 
1. Episodios de EDA en los últimos seis meses: Se asume que tal antecedente puede ser generador de desplome nutricional.

2. Nivel de pobreza: Se midió con una variable "proxi” denominada "hacinamiento crítico" (más de tres habitantes por dormitorio).

3. Edad: Se categorizó en 2 grupos: Menores de un año y mayores de un año.

Para el análisis estadístico se utilizó un modelo que plantea que los perímetros braquial y cefálico, tomados como dos variables dependientes, se relacionan con haber tenido EDA en los últimos 6 meses, con la pobreza y con la edad. En dicho modelo deben considerarse las interacciones entre estas variables. Si bien el modelo real supone relaciones bidireccionales, en esta primera fase de análisis de plantea una relación unidireccional (EDA - POBREZA - DNT). Para desarrollar dicho modelo se ejecutó un Análisis de Varianza Multivariado (MANOVA) 2 × 2 × 2 con dos variables dependientes para medir el estado nutricional, utilizando un modelo de efectos fijos, de 3 vías, con interacción. Este método es una generalización del ANOVA, a una situación en la que hay varias variables dependientes (VDs) (33), y mide si las diferencias en las medias entre grupos en relación con una combinación de variables dependientes pueden ocurrir por azar. El MANOVA funciona mejor con VDs moderadamente correlacionadas (34). A pesar de ser un método cuyo análisis es más complicado que el del ANOVA, ya que supone un análisis más riguroso de premisas, se seleccionó sobre el de ANOVAS múltiples debido a las siguientes consideraciones (35):

1. Al medir varias VDs se aumenta la oportunidad de encontrar qué es lo que cambia como resultado de los diferentes tratamientos y sus interacciones.

2. Se protege de inflar el error tipo I debido a comparaciones múltiples si se usan varios ANOVAS.

3. Pueden revelarse diferencias que no son evidentes en ANOVAS separados.

Si se demuestra que la interacción entre los diferentes factores es significativa y positiva pierde todo sentido la interpretación puntual de cada uno de los efectos principales de los factores (36). Para el análisis del modelo multivariado se utilizó el programa MANOVA de SPSS ${ }^{\circledR}$. La prueba de normalidad de datos multivariados se efectuó con el programa STATA ${ }^{\circledR}$. Para el análisis de los datos se efectuó un ajuste secuencial para no ortogonalidad.

RESULTADOS 
Se analizaron 2845 mediciones. En dicha muestra el 13,6 \% de los niños menores de 6 meses presentaron al menos un episodio de EDA en los 6 meses previos a la medición (IC ${ }_{95 \%}$ : 12,3 a 14,8). El 15,2 \% viven en hacinamiento (IC $95 \%: 13,9$ a 16,5$)$. El 12,6 \% de los niños de la muestra tienen menos de 1 año de edad (IC $95 \%$ : 11,4 a 13,9).

La media del perímetro braquial fue 16,2 centímetros (IC $95 \%$ : 16,0 a 16,32) y la de perímetro cefálico 48,1 cm ( IC $95 \%$ : 48,00 a 48,29).

Los anteriores estimadores de parámetros poblacionales se calcularon haciendo el ajuste correspondiente al método de muestreo empleado (37).

Para el análisis multivariado el orden de entrada de las variables independientes fue EDA - hacinamiento crítico - edad. Las dos variables dependientes mostraron una correlación baja $(\mathrm{r}=0.3)$ y distribución simétrica. Usando el método de Hadi (38), no se detectaron valores extremos multivariados. Mediante el uso de herramientas gráficas, la evaluación de las premisas de linealidad y multicolinealidad fue satisfactoria para poder ejecutar el método. La homogeneidad de las matrices de varianza - covarianza se evaluó con la prueba M de Box del programa SPSS MANOVA; aunque se descartó homogeneidad de las matrices de varianza - covarianza, de acuerdo con lo planteado por Hakstian, Roed y Lind (39) se consideró que no había influencia sobre los valores de error tipo I, dado que los mayores valores de varianza no dependían de las celdas más pequeñas.

Usando el criterio de Lambda de Wilks, en la prueba de ómnibus se encontró que las VDs combinadas eran significativamente afectadas por el antecedente de EDA en los últimos 6 meses $\left(\mathrm{F}_{(2,2824)}=9.65, \mathrm{p}=0.000\right)$, por la edad $\left(\mathrm{F}_{(2,2824)}=350.81, \mathrm{p}=0.000\right)$ y por la interacción EDA - Hacinamiento ( $\left.\mathrm{F}_{(2,2824)}=6.25 \mathrm{p}=0.000\right)$.

El modelo predice mejor la variabilidad del perímetro cefálico que la del perímetro braquial $\left(\eta_{\text {cefálico }}^{2}=0.332, \eta^{2}\right.$ braquial $\left.=0.063\right)$.

Para evaluar el impacto de cada variable independiente sobre cada VD individualmente, se efectuó un análisis de reducción de Roy-Bargmann. Este método permite controlar la tasa de error tipo I por múltiples pruebas $\mathrm{F}$, y ajustar el análisis por la presencia de correlación entre las VDs. Previamente se evaluó la homogeneidad de la regresión para los componentes del análisis. Los resultados de este análisis de reducción y los de las F univariadas son 
equivalentes a un nivel de significación de 0.01 . Los resultados se muestran en la Tabla 1.

Tabla 1. Valores F univariados y de Roy-Bargmann del modelo entre las Variables Dependientes (Perímetros Braquial y Cefálico) e Independientes (EDA, Hacinamiento, Edad)

\begin{tabular}{|c|c|c|c|}
\hline VD & $\mathrm{VI}$ & F univariada & F Roy-Bargmann \\
\hline \multirow{7}{*}{$\begin{array}{l}\text { Perímetro } \\
\text { Braquial }\end{array}$} & EDA & $13.10^{*}$ & $13.10^{\star}$ \\
\hline & Hacinamiento & 1.05 & 1.05 \\
\hline & Edad & $107.28 *$ & $107.28 *$ \\
\hline & EDA*Hacinamiento & 2.27 & 2.27 \\
\hline & $\mathrm{EDA}^{\star}$ Edad & 0.44 & 0.44 \\
\hline & Hacinamiento^Edad & 0.004 & 0.004 \\
\hline & EDA*Hacinamiento*Edad & 1.36 & 1.36 \\
\hline \multirow{7}{*}{$\begin{array}{l}\text { Perímetro } \\
\text { Cefálico }\end{array}$} & EDA & 2.26 & 6.18 \\
\hline & Hacinamiento & 0.48 & 0.20 \\
\hline & Edad & $686.68^{\star}$ & $572.64^{*}$ \\
\hline & EDA*Hacinamiento & $12.08^{*}$ & $10.22^{*}$ \\
\hline & EDA*Edad & 0.004 & 0.05 \\
\hline & Hacinamiento^Edad & 0.03 & 0.03 \\
\hline & EDA*Hacinamiento*Edad & 3.17 & 2.36 \\
\hline
\end{tabular}

Se encontró que el perímetro braquial tiene una contribución única para predecir el antecedente de haber tenido EDA en los últimos 6 meses (F de Roy-Bargmann 1,1=13.1 p=0.000). De tal manera, quienes tienen antecedente de EDA en los últimos seis meses tienen perímetros braquiales menores (los valores marginales del perímetro braquial ajustado por la edad categorizada son 15,2 cm, en quienes tienen antecedente de EDA y 15,6 cm para los que no tienen dicho antecedente).

Las dos VDs contribuyen a diferenciar entre los dos grupos de edad (F de Roy-Bargmann1,1=572.64 y 107.28 p=0.000).

El perímetro cefálico tiene una contribución única para predecir el tener simultáneamente EDA en los seis meses previos y vivir en hacinamiento ( $\mathrm{F}$ de Roy-Bargmann 1, $1=10.22 \mathrm{p}=0.001$ ). Los valores de las medias marginales del perímetro cefálico ajustadas por el efecto de la edad se ilustran en la Tabla 2. 
Tabla 2. Valores en las medidas marginales del perímetro cefálico según la existencia de hacinamiento y algún episodio de EDA en los últimos 6 meses, ajustadas por edad

\begin{tabular}{lccc} 
& & \multicolumn{3}{c}{ Hacinamiento Crítico } \\
& & SI & NO \\
\hline EDA en los & SI & $46,3 \mathrm{~cm}$ & $47,4 \mathrm{~cm}$ \\
\cline { 2 - 4 } & & & \\
\cline { 2 - 4 } & NO & $47,3 \mathrm{~cm}$ & $46,9 \mathrm{~cm}$
\end{tabular}

De acuerdo con estos datos, quienes simultáneamente tienen antecedentes de EDA en los 6 meses previos y viven en hacinamiento, presentan los menores perímetros cefálicos. Esto se ilustra en el gráfico de interacción de la Figura 1.

Figura 1. Interacción para relación entre perímetro cefálico y hacinamiento crítico según la existencia de EDA

$\bullet$ EDA S

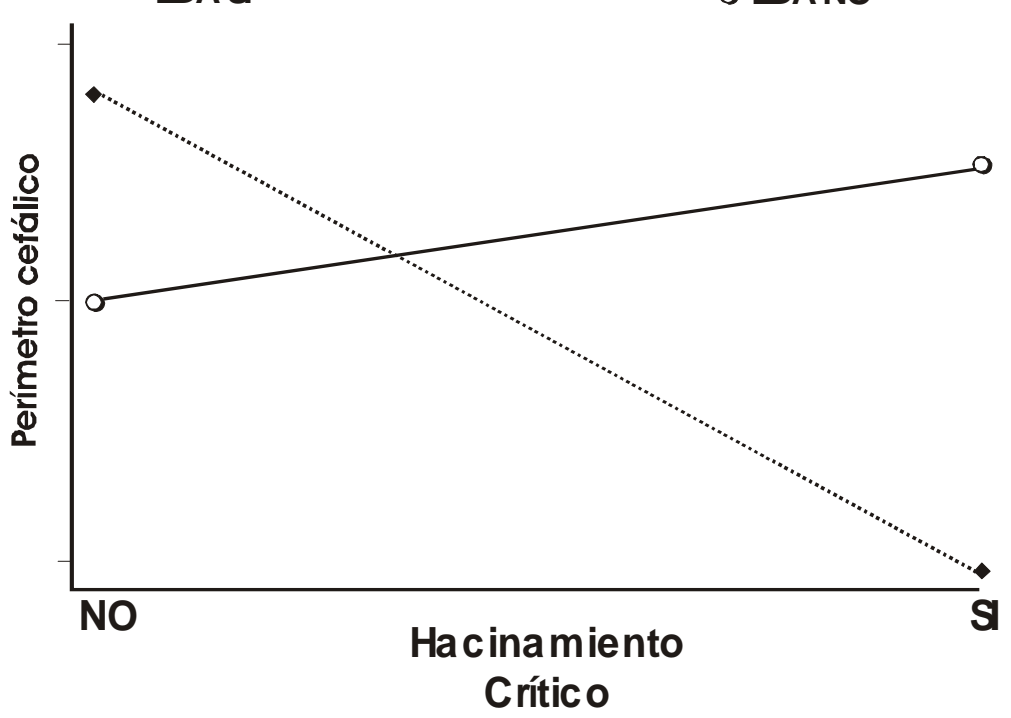

EDA SI: Al menos un episodio de Enfermedad Diarreica Aguda en los últimos 6 meses EDA NO: Sin un episodio de Enfermedad Diarreica Aguda en los últimos 6 meses 


\section{DISCUSIÓN}

El presente estudio analiza una relación documentada entre EDA, pobreza y DNT. Se efectuó el análisis estadístico mediante MANOVA, teniendo en cuenta la correlación existente entre perímetro braquial y cefálico. Dentro del análisis se controló por la variable edad, dada la obvia relación entre esta variable y las medidas antropométricas.

Se encontró que el impacto de un episodio de EDA se refleja más en el perímetro braquial. Esto puede sugerir que el brazo se tome como un indicador útil de desplome nutricional. Podría estandarizarse esta medida de tal forma que sea tomada al inicio y al final de un episodio de EDA, como forma de establecer el impacto nutricional del episodio y efectuar el seguimiento de la recuperación nutricional del niño. En síntesis, el perímetro braquial puede ser un indicador de DNT aguda.

Asumiendo la validez del hacinamiento como un proxi de pobreza, los niños que simultáneamente tienen esta condición y un episodio de diarrea en los últimos seis meses tienen perímetros cefálicos menores. Esto puede estar reflejando un grupo de menores en los que la pobreza, junto con la presencia de enfermedades infecciosas estén produciendo un impacto más generalizado sobre el desarrollo pondo estatural, o, vista la situación desde otra direccionalidad, niños desnutridos por la pobreza en quienes se presentan con mayor frecuencia este tipo de enfermedades. En este caso podría plantearse que el perímetro cefálico sea un indicador de DNT crónica.

En estudios poblacionales es difícil medir “pobreza” y "DNT”. En estas circunstancias no se evalúan casos extremos, que son los que asisten a consulta y sobre los cuales, constructos tales como la desnutrición son más fáciles de medir. El presente estudio muestra que el papel aislado de mediciones tales como los perímetros cefálico y braquial es cuestionable dado que, a nivel de estudios poblacionales, la diferencia entre "enfermos" y "sanos" es muy pequeña (solo algunos milímetros), lo cual compromete de manera importante sus características como prueba diagnóstica en tal escenario. En el caso del Perímetro Braquial, la diferencia existente entre aquellos con y sin EDA reciente es de tan sólo cuatro milímetros, con un valor para los casos de EDA reciente de 15,2 cm. En aquellos con hacinamiento y EDA reciente, el perímetro cefálico resultó siete milímetros menor que en aquellos sin hacinamiento y sin EDA reciente, con un valor para estos últimos de 46,9 $\mathrm{cm}$. Una recomendación surgida del presente trabajo es que se desarrollen instrumentos e indicadores para medir de manera válida y confiable condi- 
ciones como la desnutrición o la pobreza, o que se generen índices de riesgo que evalúen el papel simultáneo de múltiples determinantes.

Como puede verse de los resultados arrojados por este estudio, la relación entre las variables analizadas no puede establecerse en un solo sentido: DNT antecede a EDA, EDA genera desplome nutricional, la DNT a largo plazo produce individuos menos aptos para adaptarse y por ende con mayor riesgo de vivir en situación de pobreza. En un plano bidimensional, la relación podría expresarse gráficamente como lo muestra la Figura 2.

Figura 2. Relación bidimensional entre EDA y DNT

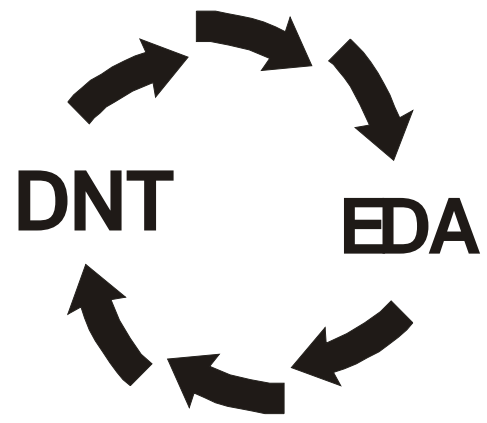

Incorporando una dimensión adicional, la pobreza, esta relación puede expresarse como lo muestra la Figura 3.

Figura 3. Relación multidimensional DNT - EDA - POBREZA

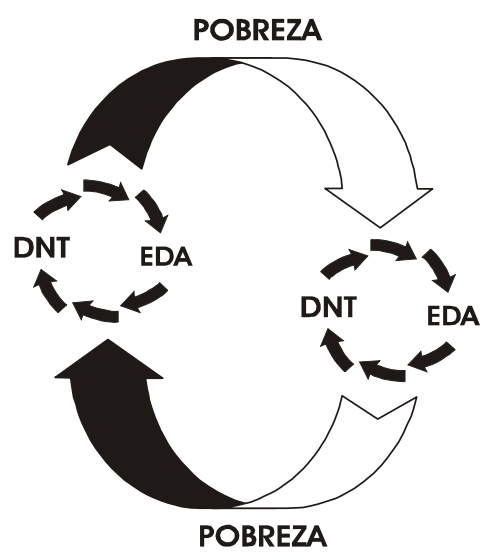

Puede incluso plantearse, que la relación circular, tal como lo sugiere este estudio, sea definida como un subtipo o síndrome particular: El síndrome 
Pobreza-desnutrición-EDA. Para la definición de tal estructura sindromática, es necesario que los tipos de relaciones entre grupos sintomáticos o dominios se estudien con técnicas estadísticas específicas que permitan analizar características de variables latentes. Dentro de dichas técnicas se encuentran las ecuaciones estructurales de análisis causal (40).

Agradecimientos. Al profesor Jaime Becerra (q.e.p.d), por su amistad y sus enseñanzas. A la Secretaría de Salud de Bogotá por la base de datos con la que se efectuó este estudio.

\section{REFERENCIAS}

1. Javaid N, Haschke F, Pietschnig B, et al. Interactions between infections, malnutrition and iron nutritional status in Pakistani Infants. A Longitudinal study. Acta Paediatr Scan Suppl 1991;374:141 -50.

2. Araya M, Espinoza J, Brunser O, Pacheco I, Cruchet S. Application of the model for predicting morbidity in children from low socioeconomic level. Rev Med Chil 1992;120(3): 342-8.

3. Punyaratabandhu P, Vathanophas K, Varavithya W, Shangchai R, et al. Childhood diarrhoea in a low-income urban community in Bangkok: incidence, clinical features, and child caretaker's behaviours. J Diarrhoeal Dis Res 1991; 9(3):244-9.

4. Benguigui Y, López FJ, Sdhmunis G, Yunes J. Eds. Infecciones Respiratorias en niños-Washington, DC.:OPS; c1997. Xxv, 496p.-(OPS. Serie HCT/ AIEPI-1).

5. Programme for the control of Diarrhoeal Disease. A manual for the treatment of diarrhoeal. Geneve: World Health Organization; 1990. WHO/CDD/ SER/80.2 Rev 1990.

6. OMS. Clinical management of Acute Diarrhea. WHO/DDC:79.3.

7. Echeverry J, Rojas E. Enfermedad diarreica infecciosa. En: Álvarez E, Paláu JM (Ed). Infecciones en Pediatría, Prevención, diagnóstico y tratamiento. $2^{\circ}$ Edición. Bogotá: McGraw Hill Interamericana; 1997. p. 289-306.

8. Hoyle B, Yunus M, Chen LC. Breast feeding and food intake among children with acute diarrhoeal disease. Am J Clin Nutr. 1980;33:2365-71.

9. Departamento Nacional de Estadística, DANE. Boletín DE Estadística, No 429; diciembre de 1988.

10. Departamento Administrativo Nacional de Estadísticas, Departamwento Nacional de Planeación, PDUD, UNICEF. La pobreza en Colombia, Tomo I,. 1989. p. 458.

11. Universidad Nacional de Colombia, FESCOL, CINEP. Equidad en la política social en Colombia I. 1999.

12. Ramos-Galván R. Significado y empleo de las referencias somatométricas de peso y talla en la práctica pediátrica y epidemiológica. Bol Med Hosp In Mex 1992;49(6):321-24. 
13. Viteri FE. Protein energy malnutrition. In: Walker WA, Durie PR, Hamilton JR, et al, (Ed.). Pediatric Gastrointestinal Disease, Pathophisiology , diagnosis, management, vol 2. Philadelphia: B.C.Decker; 1991. p. 1596 -1611.

14. Morley DC. Measles in the developing world. Proc Roy Soc Med. 1974;67:1112-15.

15. Rosenberg IH. Solomons NW. Malabsortion associated with diarrhea and intestinal infections. Am J Clin Nutr. 1972; 30:1248-53.

16. Whithead RG. Protein and energy requirements of young children living in the developing countries to allow for cach-up growth after infections. Am J Clin Nutr 1977;30:1545-47.

17. Rothman KJ, Greenland S. Causation and Causal Inference. In: Rothman KJ, Greenland S, (Eds). Modern Epidemiology. $2^{\circ}$ Edition. Philadelphia, Pa: Lippincott-Raven Publishers; 1998. p. 7- 28.

18. Martorell R, Yarbrough C, Yarbrough S, Klein RF. The impact of ordinary illnesses on the dietary intakes of malnourished children. Am J Clin Nutr 1980; 33:345-50.

19. Rowland MG, Cole TJ, Whithead RGA. A quantitative study into the role of infection in determining nutritional status in Gambian village children. $\mathrm{Br} \mathrm{J}$ Nutr. 1977;441-50.

20. Hirschhorn N, Denny KM. Oral glucose electrolyte therapy for diarrhea: A means to maintain or improve nutrition. Am J Clin Nutr. 1975;28:189-92.

21. Black RE, Chel LC, Harkavy O, Rahaman MM, Rowland MGM. Prevention and control of the diarrhoeal disease. In: Chen LC, Scrimshaw NS (Eds). Diarrhea and malnutrition: Interactions, mechanisms and interventions. New York: Plenum Press; 1983:297 -303.

22. Chung AW, Viscorová B. The effect of early oral feeding versus early oral starvation on the course of the infantile diarrhea. J Pediatr 1948;33:14 - 20.

23. Echeverry J, Ardila E. Pruebas diagnósticas y proceso diagnóstico. En: Ardila E, Sánchez R, Echeverry J, (Ed.). Estrategias de investigación en medicina clínica. Bogotá: Editorial el Manual Moderno; 2001. p. 135 - 68.

24. Fomon SJ, Nelson SE. Tamaño y Crecimiento. En: Fomon SJ (Ed.). Nutrición del lactante. Bogotá: Mosby/Doyma Libros; 1995. p. 36 -83.

25. Mclaren DS, Read WWC. Classification of nutritional status in early childhood. Lancet 1972;2:146-7.

26. Waterlow J. Classification and definition of protein-calorie malnutrition. BMJ 1972;2:566-9.

27. Wright J, Ashemburg CA, Whitaker RC. Comparison of methods to categorize under nutrition in children. J Pediatr. 1994; 124:944 - 6.

28. National Centre of Health Statistics: MCHS Growth Curves for children 0-18 years. United States, Vital and Health Statistics, Series 11. N.165. Washington, DC, Health Resources Administration, US Government Printing Office, 1977.

29. Peterson K, Chen L. Defining undernutrition for public health purposes in the United States. J Nutr 1990; 120:933-42. 
30. Besada S. Desnutrición. En: Rojas C, Guerrero R. Nutrición clínica y gastroenterología pediátrica. Bogotá: Editorial Médica Panamericana; 1999. pp.141 58.

31. Marín MA, González MC, Alonso ME, Beltrán M. Circunferencia de brazo como indicador de riesgo de desnutrición en preescolares. Salud Publica Mex 1993; 35: 667-672.

32. Donabedian A. (1966). Evaluación de la Calidad de atención médica. En: Whie KL, Frenk J, Ordóñez, y cols. (Ed.). Investigaciones sobre servicios de salud: Una antología. Washington: OPS; 1992. p. 382-404.

33. Gnanadesikan R. Methods for statistical data analysis of multivariate observations. New York: John Wiley and Sons, Inc.; 1997. p.256.

34. Tabachnick BG, Fidell LS. Using multivariate statistics. 3rd ed. Northridge: Harper Collins Publishers Inc.; 1996. p. 406.

35. Bray JH, Maxwell SE. Multivariate analysis of variance. Newbury Park: SAGE Publications; 1985.

36. Arnau J. Diseños experimentales multivariables. Madrid: Alianza Editorial; 1990. p.280.

37. Levy PS, Lemeshow S. Sampling of populations. Methods and applications. 3rd edition. New York: John Wiley and Sons, Inc.;1999. p. 145-189.

38. Hadi AS. Identifying multiple outliers in multivariate data. Journal of the Royal Statistical Society 1992;54:761-771.

39. Hakstian AR, Roed JC, Lind JC. Two-sample T2 procedure and the assumption of homogeneous covariance matrices. Psychological Bulletin 1979; 86:1255-1263.

40. Maruyama GM. Basics of structural equation modeling. Thousand Oaks: Sage Publications; 1998. p. 3-14. 